\title{
Severe hypoglycaemia under abemaciclib administration in a patient with breast cancer: A case report
}

\author{
TATSUO HORIE ${ }^{1}$, TSUNETAKA KIJIMA ${ }^{2}$, MINEKAZU YAMAGUCHI ${ }^{2,3}$, SATOSHI HONDA ${ }^{2}$, MIYAKO HORIE ${ }^{1}$, \\ KAZUNARI ISHITOBI $^{3}$, SHINGO YAMAGATA ${ }^{2}$, SHIGERU SAKANO $^{3}$ and KAZUTAKA KUROKOHCHI ${ }^{2,4}$ \\ ${ }^{1}$ Department of Pharmacy, Oda Municipal Hospital, Oda, Shimane 694-0063; ${ }^{2}$ Department of General Medicine, \\ Shimane University Faculty of Medicine, Oda General Medicine Education Center; \\ ${ }^{3}$ Department of Surgery, Oda Municipal Hospital, Oda, Shimane 694-0063; \\ ${ }^{4}$ Department of General Medicine, Meiwa Hospital, Nishinomiya, Hyogo 663-8186, Japan
}

Received February 12, 2020; Accepted January 11, 2021

DOI: $10.3892 / \mathrm{mco} .2021 .2223$

\begin{abstract}
The current study reports the case of an 80-year-old woman who experienced severe hypoglycaemia after abemaciclib administration, with a recovery time of $\sim 46 \mathrm{~h}$. Abemaciclib is a cyclin-dependent kinase 4 and $6(\mathrm{CDK} 4 / 6)$ inhibitor that is used to treat metastatic breast cancer. A side effect of abemaciclib administration is an increase in creatinine levels. The half-life $\left(t_{1 / 2}\right)$ of $150 \mathrm{mg}$ abemaciclib in patients with breast cancer was reported to be $17.5 \mathrm{~h}$ (nearly lower limit), and the time to reach $\mathrm{C}_{\max }$ was $\sim 5 \mathrm{~h}\left(\mathrm{~T}_{\max }, 4-6 \mathrm{~h}\right)$. Therefore, the total time to reach half the maximum blood concentration after abemaciclib administration is $\sim 24 \mathrm{~h}\left(\mathrm{~T}_{\max }+\mathrm{t}_{1 / 2}=5+17.5=22.5 \mathrm{~h}\right)$. As abemaciclib is administered twice daily, a considerable amount $\left(\mathrm{C}_{\max }=123 \mathrm{ng} / \mathrm{ml}\right)$ may persist in the blood following the initial dose. Upon repeated administration, the blood abemaciclib concentration in patients with metastatic liver tumours might increase, although their liver function remains normal. The patient described in the current study had a creatinine level of $1.05 \mathrm{mg} / \mathrm{dl}$ at the start of abemaciclib administration. At the time of emergency hospitalisation (on day 5 of abemaciclib administration), the creatinine level was $1.40 \mathrm{mg} / \mathrm{dl}$; however, dehydration was not observed. The patient had been administered the same dose of glimepiride for $>1$ year and had not experienced hypoglycaemia previously. It can be speculated that the increase in blood creatinine level had some effect on glimepiride metabolism. It is thought that administered abemaciclib enhances metabolic delay in the blood in the same way as in patients with impaired liver function, and as a result, the creatinine level increases in patients with liver metastases. This causes a decrease in renal function, which in turn results in an increase in blood concentration of glimepiride, consequently
\end{abstract}

Correspondence to: Mr. Tatsuo Horie, Department of Pharmacy, Oda Municipal Hospital, 1428-3 Oda-Yosinaga, Oda, Shimane 694-0063, Japan

E-mail: yakuzai@ohda-hp.ohda.shimane.jp

Key words: abemaciclib, creatinine, cyclin-dependent kinase 4 and 6, diabetes, protein-binding, renal dysfunction, severe hypoglycaemia leading to severe hypoglycaemia. Therefore, clinicians must be careful when using abemaciclib in patients with liver metastases, diabetes and poor renal function.

\section{Introduction}

Cancer is not the leading cause of death worldwide, but cancer associated mortality has increased in recent years $(1,2)$. With stratification by income, it has been indicated that cancer mortality rates are steadily increasing in high-income countries compared with low-income countries $(1,2)$. According to data from the International Agency for Research on Cancer (IARC) World Cancer Statistics GLOBOCAN, breast cancer is the most frequent cancer in women, accounted for $24 \%$ of newly diagnosed cancers in 2018 and $15 \%$ of cancer deaths, and these rates are expected to increase in the future $(1,2)$. In addition, it is considered that the worldwide population will be aging in the future (3). Moreover, in 2019, a total of 463 million people were estimated to be living with diabetes (4), representing $9.3 \%$ of the global adult population (20-79 years), with a prevalence of $9.0 \%$ in women and $9.6 \%$ in men. The number of people living with diabetes is projected to increase by $25 \%$ to 578 million by 2030 and by $51 \%$ to 700 million by 2045 globally (4). The morbidity and mortality associated with aging, diabetes, and breast cancer are also very relevant concerns for the Japanese population. Therefore, novel therapeutic drugs for breast cancer and diabetes are continuously being developed; However, with increasing numbers of patients with comorbidities, the interactions, side effects, and adverse events of these therapeutic drugs are becoming increasingly more complicated. Under these circumstances, it is important to provide safe and secure medical care to elderly patients in particular, and it is expected that the need for a team approach to medical care consisting of many specialists, including doctors and pharmacists, will become even more important in the future. The current reports describes a case of severe hypoglycaemia in a patients with breast cancer that persisted for $>24 \mathrm{~h}$ after the administration of abemaciclib, an antitumor agent and dual inhibitor of cyclin-dependent kinases 4 and 6 (CDK4/6). In November 2018, Japan approved the use of abemaciclib for the treatment of hormone 
receptor-positive and HER2-negative advanced and metastatic breast cancer (5-7). Currently, to the best of our knowledge, there have been no detailed reports regarding cases of severe hypoglycaemia associated with the use of abemaciclib to date.

\section{Case report}

In March 2013, an 80-year-old woman who had developed multiple bone, liver, and ovarian metastases from right breast cancer (ER+: 90\%, PgR8+: 90\%, HER2-) was administered letrozole orally and denosumab subcutaneously. In June 2015, letrozole treatment was replaced with fulvestrant (Ful) owing to progressive disease (PD). In February 2018, haemorrhagic advanced breast cancer [Br+AX (level 1), T4N1M1, pT4bN1MX, stage IV] mastectomy was performed. Administration of Ful was continued thereafter. In July 2018, despite treatment with Ful, the patient experienced PD; therefore, bevacizumab plus paclitaxel therapy was initiated. In March 2019, due to PD as detected by computed tomography, abemaciclib plus Ful therapy was initiated. The pateent's glycated haemoglobin (HbA1c) level was $5.9 \%$ 3 weeks before the initiation of abemaciclib treatment, which commenced 4 days before hospitalisation (-day 4) at an oral dose of $150 \mathrm{mg}$, twice daily. However, adverse events such as poor physical condition, abdominal pain, and diarrhoea occurred on the second day post abemaciclib treatment (-day 3). Therefore, the dose of abemaciclib was reduced to $100 \mathrm{mg}$, twice a day, from -day 1 to hospitalisation. The patient reported tremors and insomnia that same night. Furthermore, on the following day (day 1: Day of hospitalisation), she reported subjective symptoms of diplopia, but she was able to eat a full meal three times that day. At $\sim 21: 45 \mathrm{~h}$, the patient's family noticed that the patient was unfocused, with impaired speech; therefore, they contacted the emergency department of the hospital 5 min later. The patient was rushed to the hospital by her family at 22:40 h. As she was in the supine position in the back seat of the car, the staff transferred her to a stretcher with full assistance. The patient responded slightly to our call, but her level of consciousness declined, and she was unable to speak and focus. Her breathing was normal and there were no abnormal laboratory findings in her chest and abdomen during emergency room (ER) observation. The laboratory findings in the emergency outpatient clinic revealed extremely low blood glucose level of $24 \mathrm{mg} / \mathrm{dl}$ (Fig. 1A), indicating hypoglycaemia (Tables I and II). Moreover, renal dysfunction was indicated as her serum creatinine level was $1.40 \mathrm{mg} / \mathrm{dl}$.

Emergency outpatient clinical course: In the ER, the patient was not able to focus her eyes and she was unable to answer our call. As the blood tests revealed hypoglycaemia, we administered $40 \mathrm{ml}$ of $40 \%$ glucose solution intravenously. The patient's level of consciousness returned to normal immediately after the injection. She could make eye contact and converse with us. After $60 \mathrm{~min}$, at 23:40 h, her blood glucose level increased to $91 \mathrm{mg} / \mathrm{dl}$ (Fig. 1B), and she was able to urinate independently in the toilet and move to a wheelchair stably. The patient did not remember the time she arrived at the hospital because of hypoglycaemia. In the ER, we asked her family about her medical history and medications, including whether she had diabetes. The patient had no history
Table I. Observations at admission.

\begin{tabular}{ll}
\hline Clinical characteristics & \multicolumn{1}{c}{ Value } \\
\hline Body height & $154.5 \mathrm{~cm}$ \\
Body-weight (BMI) & $57.2 \mathrm{~kg}\left(23.96 \mathrm{~kg} / \mathrm{m}^{2}\right)$ \\
Body temperature & $34.5^{\circ} \mathrm{C}$ \\
Blood pressure & $118 / 52 \mathrm{mmHg}$ \\
Pulse & $67 / \mathrm{min}$ \\
Oxygen saturation & $94 \%$ \\
Consciousness level (GCS) & $13=\mathrm{E} 4+\mathrm{V} 4+\mathrm{M} 5$
\end{tabular}

Table II. Physiological data at the time of hospitalization.

\begin{tabular}{lc}
\hline Blood and biochemical tests & Value \\
\hline $\mathrm{AST}$ & $29 \mathrm{IU} / \mathrm{l}$ \\
$\mathrm{ALT}$ & $21 \mathrm{IU} / \mathrm{l}$ \\
$\mathrm{BUN}$ & $20.4 \mathrm{mg} / \mathrm{dl}$ \\
$\mathrm{Cr}$ & $1.4 \mathrm{mg} / \mathrm{dl}$ \\
$\mathrm{CK}$ & $108 \mathrm{IU} / \mathrm{l}$ \\
$\mathrm{Na}$ & $141 \mathrm{mEq} / \mathrm{l}$ \\
$\mathrm{Cl}$ & $108 \mathrm{mEq} / 1$ \\
$\mathrm{Ca}$ & $8.6 \mathrm{mEq} / 1$ \\
$\mathrm{BS}$ & $24 \mathrm{mg} / \mathrm{dl}$ \\
$\mathrm{WBC}$ & $66.1 \times 10^{3} / \mu 1$ \\
$\mathrm{RBC}$ & $342 \times 10^{4} / \mu 1$ \\
$\mathrm{Hb}$ & $108 \mathrm{~g} / \mathrm{dl}$ \\
$\mathrm{Ht}$ & $31.40 \%$ \\
$\mathrm{PLT}$ & $12.7 \times 10^{3} / \mu 1$ \\
$\mathrm{Neut}$ & $55.2 \times 10^{2} / \mu 1$ \\
$\mathrm{Lymph}$ & $7.9 \times 10^{2} / \mu 1$ \\
\hline
\end{tabular}

AST, aspartate aminotransferase; ALT, alanine aminotransferase; BUN, blood urea nitrogen; CK, creatine kinase; BS, blood sugar; WBC, white blood cell; RBC, red blood cell; PLT, platelet.

of receiving antidiabetic treatment at other hospitals and had no experience of hypoglycaemia while receiving antidiabetic drugs for more than a year (Table III). Additionally, she had eaten all her meals on the day of hospitalisation (day 1). She had self-managed abemaciclib medication (Verzenio), which was recorded in the 'Verzenio Diary'. We were able to confirm that there was no overdose of glimepiride (Gli) tablets from the patient's remaining medication.

The patient was admitted to the surgical department on the same day for hypoglycaemia treatment and follow-up. All outpatient prescription drugs were discontinued at the time of hospitalisation. The patient's clinical course after admission is shown in Fig. 1. She was conscious at 00:10 h on the second day of admission. As she complained of hunger, she was provided a banana and tea by her family. We did not detect symptoms such as diplopia, numbness, and cold sweats. The patient seemed to have independently used the toilet during the night without the aid of a nurse, as we detected a large quantity of urine in a 


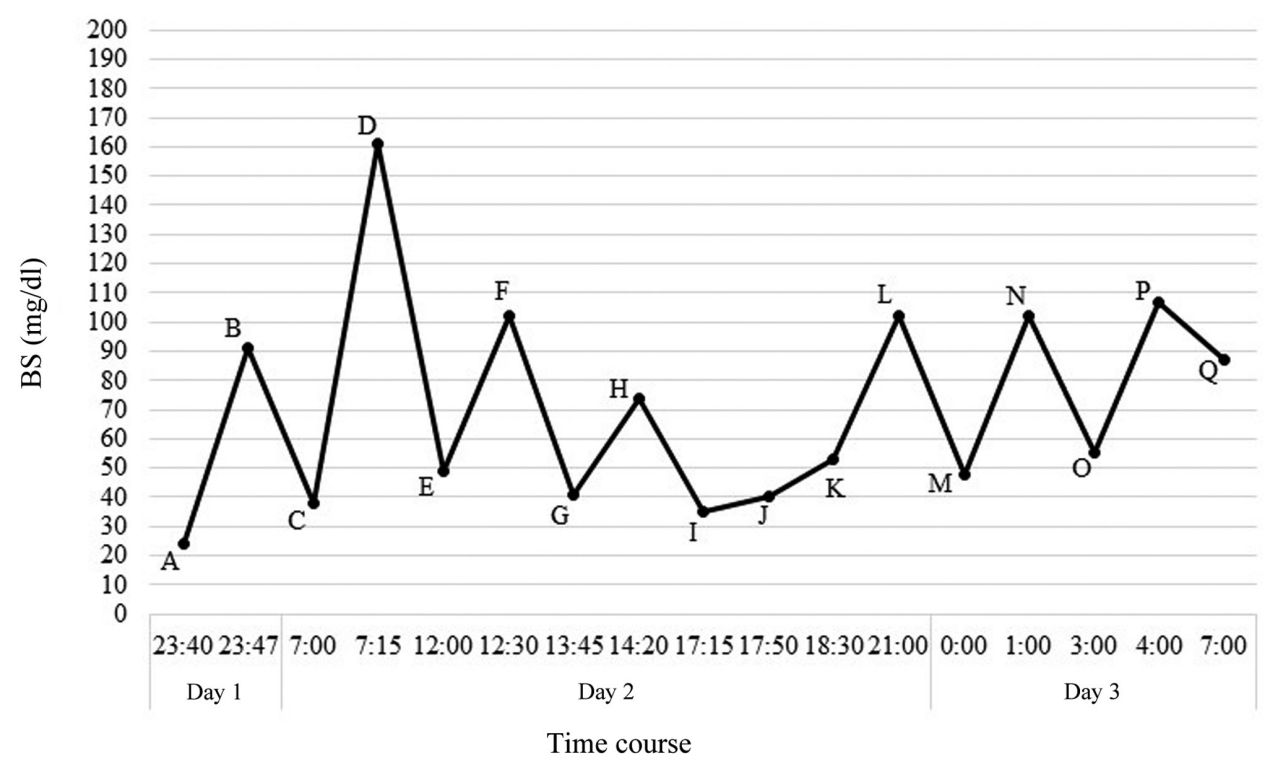

Figure 1. Patient's time course of blood glucose level and treatment after admission (A) day 1, 23:40 h, measurement of blood glucose and i.v. administration of $20 \mathrm{ml}$ of $40 \%$ glucose solution; (B) day 1,23:47 h, measurement of blood glucose, and the patient consumed a midnight snack; (C) day 2, 7:00 h, measurement of blood glucose and i.v. administration of $40 \mathrm{ml}$ of $40 \%$ glucose solution; (D) day 2, 7:15 h, measurement of blood glucose, and the patient consumed breakfast; (E) day 2, 12:00 h, measurement of blood glucose and i.v. administration of $20 \mathrm{ml}$ of $40 \%$ glucose; (F) day 2, 12:30 h, measurement of blood glucose, and the patient consumed lunch; (G) day 2, 13:45 h, measurement of blood glucose and oral administration of $10 \mathrm{~g}$ glucose; (H) day 2, 14:20 h, measurement of blood glucose; (I) day 2, 17:15 h, measurement of blood glucose and i.v. administration of $20 \mathrm{ml}$ of $40 \%$ glucose solution; (J) day 2, 17:50 h, measurement of blood glucose and i.v. administration of $20 \mathrm{ml}$ of $40 \%$ glucose; the patient consumed dinner; (K) day 2, 18:30 h, measurement of blood glucose and oral administration of $10 \mathrm{~g}$ glucose; (L) day 2, 21:00 h, measurement of blood glucose; (M) day 3, 0:00 h, measurement of blood glucose and oral administration of $10 \mathrm{~g}$ glucose; $(\mathrm{N})$ day $3,1: 00 \mathrm{~h}$, measurement of blood glucose; $(\mathrm{O})$ day 3, 3:00 h, measurement of blood glucose and oral administration of $10 \mathrm{~g}$ glucose; (P) day 3, 4:00 h, measurement of blood glucose; (Q) day 3, 7:00 h, measurement of blood glucose. BS, blood sugar.

Table III. Prescription drugs: Daily dose.

\begin{tabular}{|c|c|}
\hline Oral medication & Dose \\
\hline Abemaciclib (100 mg) & Twice (after breakfast and dinner) \\
\hline Glimepiride (1 mg) & Twice (after breakfast and dinner) \\
\hline $\begin{array}{l}\text { Loxoprofen sodium } \\
\text { hydrate }(60 \mathrm{mg})\end{array}$ & Twice (after breakfast and dinner) \\
\hline Rebamipide (100 mg) & Twice (after breakfast and dinner) \\
\hline Doxazosin mesylate (2 mg) & Twice (after breakfast and dinner) \\
\hline Valsartan $(80 \mathrm{mg})$ & Once after breakfast \\
\hline Amlodipine besilate (5 mg) & Once after breakfast \\
\hline Pravastatin sodium (10 mg) & Once after dinner \\
\hline $\begin{array}{l}\text { Loperamide hydrochloride } \\
\text { (1 mg) }\end{array}$ & $\begin{array}{l}\text { Up to } 3 \text { times a day in case } \\
\text { of diarrhoea }\end{array}$ \\
\hline Brotizolam $(0.25 \mathrm{mg})$ & Before sleeping in case of insomnia \\
\hline Indomethacin patch & Topical \\
\hline
\end{tabular}

portable toilet. The patient was asleep during the nurse's patrol. At 7:00 h, during the nurse's patrol, the patient was changing clothes on the bed, but she did not respond to the nurse's calling; moreover, she could not focus her eyes. Her blood glucose level was $38 \mathrm{mg} / \mathrm{dl}$ (Fig. 1C); thus, we immediately injected $40 \mathrm{ml}$ of $40 \%$ glucose solution intravenously. Shortly after, her blood glucose level increased to $161 \mathrm{mg} / \mathrm{dl}$ (Fig. 1D), and her consciousness level returned to normal. She could maintain eye contact and we could converse with her. However, the patient did not remember any of her hypoglycaemic events. Immediately after the intravenous injection of glucose solution and oral glucose intake, her blood glucose levels increased and her consciousness improved, but the blood glucose levels later dropped back to $30-50 \mathrm{mg} / \mathrm{dl}$. This hypoglycaemic event repeated until the third day post-admission. On all 3 days in the surgical department, the patient ate all of her meals (breakfast, lunch, and dinner). At 7:00 h on the third day of admission, her blood glucose level was $87 \mathrm{mg} / \mathrm{dl}$, indicating no hypoglycaemia (Fig. 1Q). The patient's blood glucose level was maintained over $80 \mathrm{mg} / \mathrm{dl}$, and there was no relapse of hypoglycaemia. Her immunoreactive insulin level was normal at $5.56 \mu \mathrm{IU} / \mathrm{ml}$. In summary, the total administered glucose content from admission to recovery of severe hypoglycaemia was $48 \mathrm{~g}$ administered intravenously and $40 \mathrm{~g}$ administered orally, plus a regular meal of $1,600 \mathrm{kcal} /$ day and a banana. Finally, the time required to recover from severe hypoglycaemia was $\sim 46 \mathrm{~h}$. Summary of the treatment (Table IV).

\section{Discussion}

We present a case report of severe hypoglycaemia under abemaciclib administration. When the patient arrived at our hospital, she had taken prescription medicines, including abemaciclib, after a full portion of dinner. We confirmed with the family regarding the absence of any overlapping medications. In this case (from -day 4 to 0 ), no additional new medicines were administered other than abemaciclib. The patient had been using Gli and loxoprofen sodium hydrate (Lox) since a long time, and no associated hypoglycaemic events had occurred previously. Therefore, the possibility of drug (Gli and Lox)-interaction-induced hypoglycaemia was low. Gli has a high protein-binding rate according to dosage studies in 
Table IV. Summary of the treatment.

\begin{tabular}{lccc}
\hline A, Day 1 & & & \\
\hline Point & Time & Blood glucose level & Treatment \\
\hline A & $23: 40 \mathrm{~h}$ & 24 & $20 \mathrm{ml}$ of $40 \%$ glucose solution i.v. injection \\
B & $23: 47 \mathrm{~h}$ & 91 & Patient ate a midnight snack \\
\hline
\end{tabular}

B, Day 2

\begin{tabular}{lrrl}
\hline Point & Time & Blood glucose level & Treatment \\
\hline C & $7: 00 \mathrm{~h}$ & 38 & $40 \mathrm{ml}$ of $40 \%$ glucose solution i.v. injection \\
$\mathrm{D}$ & $7: 15 \mathrm{~h}$ & 161 & Patient ate breakfast \\
$\mathrm{E}$ & $12: 00 \mathrm{~h}$ & 49 & $20 \mathrm{ml}$ of $40 \%$ glucose solution i.v. injection \\
$\mathrm{F}$ & $12: 30 \mathrm{~h}$ & 102 & Patient ate lunch \\
$\mathrm{G}$ & $13: 45 \mathrm{~h}$ & 41 & Oral administration of $10 \mathrm{~g}$ \\
$\mathrm{H}$ & $14: 20 \mathrm{~h}$ & 74 & Oral administration of $10 \mathrm{~g}$ \\
$\mathrm{I}$ & $17: 15 \mathrm{~h}$ & 35 & $20 \mathrm{ml}$ of $40 \%$ glucose solution i.v. injection \\
$\mathrm{J}$ & $17: 50 \mathrm{~h}$ & 40 & $20 \mathrm{ml}$ of $40 \%$ glucose solution i.v. injection \\
$\mathrm{K}$ & $18: 30 \mathrm{~h}$ & 53 & Oral administration of $10 \mathrm{~g}$ glucose \\
$\mathrm{L}$ & $21: 00 \mathrm{~h}$ & 102 & Medical follow-up \\
\hline
\end{tabular}

\section{C, Day 3}

\begin{tabular}{lccl}
\hline Point & Time & Blood glucose level & \multicolumn{1}{c}{ Treatment } \\
\hline M & $0: 00 \mathrm{~h}$ & 48 & Oral administration of $10 \mathrm{~g}$ glucose \\
$\mathrm{N}$ & $1: 00 \mathrm{~h}$ & 102 & Medical follow-up \\
$\mathrm{O}$ & $3: 00 \mathrm{~h}$ & 55 & Oral administration of $10 \mathrm{~g}$ glucose \\
$\mathrm{P}$ & $4: 00 \mathrm{~h}$ & 107 & Medical follow-up \\
$\mathrm{Q}$ & $7: 00 \mathrm{~h}$ & 87 & Medical follow-up \\
\hline
\end{tabular}

patients with type 2 diabetes (8-11). Allylpropionic acid-based Lox also has a high protein-binding rate (12-15). Therefore, when Gli is used together with Lox, the binding of Gli to blood protein is suppressed, and the free form of Gli increases (8-11). Therefore, the combined usage of Lox with Gli may enhance the hypoglycaemic effect (16). Abemaciclib, a pyrido[2,3-d] pyrimidin-7-one inhibitor, is a selective inhibitor of CDK4 and CDK6 (17-19) that phosphorylates Rb and activates transcription factor E2F1/2. Thus, abemaciclib pushes cells into the $\mathrm{S}$ phase and triggers DNA synthesis $(20,21)$. The time to reach abemaciclib $\mathrm{C}_{\max }$ is $\sim 5 \mathrm{~h}\left(\mathrm{~T}_{\max }, 4-6 \mathrm{~h}\right)(22,23)$, and the half-life of $150 \mathrm{mg}$ of abemaciclib is $17.5 \mathrm{~h}$ (nearly lower limit: 17.4 to $38.1 \mathrm{~h})(22,23)$. Therefore, the total time to reach half the maximum blood concentration after abemaciclib administration is $\sim 24 \mathrm{~h}\left[\mathrm{~T}_{\max }+\mathrm{t}_{1 / 2}=5+17.5=22.5 \mathrm{~h}(22,23)\right]$. In other words, it takes $24 \mathrm{~h}$ for abemaciclib blood concentration to drop by half $(1 / 2)$. As abemaciclib is administered twice daily, a considerable amount of abemaciclib may persist in the blood when the second dose $(\sim 12 \mathrm{~h}$ later $)$ is administered. In patients with severe liver dysfunction, the blood concentration of this drug increases (24). With repeated dosing of abemaciclib, the blood concentration of abemaciclib in patients with metastatic liver tumours may be higher than anticipated, even with normal liver function (11). Abemaciclib has been shown to have a high human plasma protein-binding rate in in vitro studies $(5-7,22)$. Gli is primarily metabolised by the liver metabolic enzyme CYP2C9 and excreted via the kidney (urine) and liver (bile) $(8-11,25)$, while abemaciclib is metabolised by CYP3A and excreted via the liver (24). Therefore, the possibility that they influence each other's metabolism is low. Increase in blood creatinine level has been described as an adverse event of abemaciclib (5-7,24). The patient's creatinine level was $1.05 \mathrm{mg} / \mathrm{dl}$ at the start of administration (-day 4). On the fifth day of abemaciclib administration (day 1: Day of hospitalisation), the creatinine level increased to $1.40 \mathrm{mg} / \mathrm{dl}$; however, dehydration due to loose stools, diarrhoea, and other symptoms was not observed on admission.

Abemaciclib has been shown to slow metabolism in the blood of patients with impaired liver function $(5-7,24)$. In metastatic liver cancer, CYP2C9 metabolism in the liver decreases; therefore, the blood concentration of Gli increases $(11,25)$. However, our patient was administered the same dose of Gli for over 1 year and had never experienced hypoglycaemia. In addition, CYP3A4 metabolism in the liver decreases in metastatic liver cancer $(26,27)$. Consequently, the blood concentration of abemaciclib increases, which increases the creatinine level $(5-7,24)$. Furthermore, an 
increase in creatinine level suggests a decrease in renal function, which is thought to increase the blood concentration of Gli (25,28-31). Although it is unclear at present whether this case is an isolated incident of the combined biochemical and genetic profile of the patient, severe hypoglycaemia may well occurs in elderly breast cancer patients with diabetes and a history of liver metastases when abemaciclib is combined used with Gli (high protein-binding affinity) and allylpropionic acid-based Lox (high protein-binding affinity). Therefore, adverse events of the drug for these patients are likely to be worth investigating in a larger population size and those awaits further elucidation. The increase in creatinine levels following abemaciclib administration does not necessarily indicate glomerular injury. However, it is difficult to argue that the increase in creatinine levels is not related to the decrease in renal function $(5,23,32)$. Although it can not be ruled out that, the increased creatinine levels after abemaciclib treatment in patients without liver metastases, it may be lead to hyperglycemia caused by decreased water reabsorption, Low levels of $\mathrm{Ht}$ and BUN/Cre in the labo data indicated that the patient was not dehydrated at the time of transport. Although since this patient has liver metastasis, it is considered that the blood concentration of abemaciclib is increased due to the metabolic delay of abemaciclib and the blood creatinine level is increased. Increased creatinine levels suggested a decrease in renal function, which may have caused an increase in the blood concentration of Gli and the strong effect of Gli may have caused the patient's hypoglycaemia. Moreover, glucagon blood sugar increasing action is mainly due to the decomposition of hepatic glycogen, it is said that the effect of raising blood sugar can hardly be expected for liver metastasis patients (33). And in severe hypoglycemia with unconsciousness, it may be difficult to take glucose tablets or glucose powder. Based on the above, we must attend to the presence or absence of liver metastases, use of drugs that depend on renal excretion, blood glucose level should be carefully monitored, when we are using abemaciclib with diabetes patients. Then, if renal function is poor, it is necessary to immediately stop SU drugs such as Gli and switch to insulin.

\section{Acknowledgements}

Not applicable.

\section{Funding}

No funding was received.

\section{Availability of data and materials}

The datasets used and/or analyzed during the current study are available from the corresponding author on reasonable request.

\section{Authors' contributions}

$\mathrm{TH}, \mathrm{TK}$ and $\mathrm{KK}$ were involved in the conception and design of the case study; TH, MY, MH, KI and SS were involved in data acquisition; TH, MY, SH, KI, SY and SS analysed and interpreted the data. $\mathrm{TH}$ and $\mathrm{KK}$ were responsible for confirming the authenticity of the raw data. The manuscript was written by $\mathrm{TH}$ and was critically reviewed by $\mathrm{TH}, \mathrm{TK}, \mathrm{MY}, \mathrm{SH}, \mathrm{MH}$, KI, SY, SS and KK. All authors read and approved the final manuscript.

\section{Ethics approval and consent to participate}

Not applicable.

\section{Patient consent for publication}

Written informed consent was obtained from the patient for the publication of this case report.

\section{Competing interests}

The authors declare that they have no competing interests.

\section{References}

1. Jamison DT, Summers LH, Alleyne G, Arrow KJ, Berkley S, Binagwaho A, Bustreo B, Evans D, Feachem RGA, Frenk J, et al: Global health 2035: A world converging within a generation. Lancet 382: 1898-1955, 2013.

2. Ferlay J, Colombet M, Soerjomataram I, Mathers C, Parkin DM, Piñeros M, Znaor A and Bray F: Estimating the global cancer incidence and mortality in 2018: GLOBOCAN sources and methods. Int J Cancer 144: 1941-1953, 2019.

3. Raftery AE, Li N, Ševčíková H, Gerland P and Heilig GK: Bayesian probabilistic population projections for all countries. Proc Natl Acad Sci USA 109: 13915-13921, 2012.

4. Saeedi P, Petersohn I, Salpea P, Malanda B, Karuranga S, Unwin N, Colagiuri S, Guariguata L, Motala AA, Ogurtsova K, et al: Global and regional diabetes prevalence estimates for 2019 and projections for 2030 and 2045: Results from the International Diabetes Federation Diabetes Atlas, 9(th) edition. Diabetes Res Clin Pract 157: 107843, 2019.

5. Dickler MN, Tolaney SM, Rugo HS, Cortés J, Diéras V, Patt D, Wildiers H, Hudis CA, O'Shaughnessy J, Zamora E, et al: MONARCH 1, a phase II study of abemaciclib, a CDK4 and CDK6 inhibitor, as a single agent, in patients with refractory $\mathrm{HR}^{+} / \mathrm{HER} 2$ metastatic breast cancer. Clin Cancer Res 23: 5218-5224, 2017.

6. Sledge GW Jr, Toi M, Neven P, Sohn J, Inoue K, Pivot X, Burdaeva O, Okera M,Masuda N, Kaufman PA, et al: MONARCH 2: Abemaciclib, in combination with Fulvestrant in women with $\mathrm{HR}^{+} / \mathrm{HER} 2^{-}$advanced breast cancer who had progressed while receiving endocrine therapy. J Clin Oncol 35: 2875-2884, 2017.

7. Goetz MP, Toi M, Campone M, Sohn J,Paluch-Shimon S, Huober J, Park IH, Trédan O, Chen SC, Manso L, et al: MONARCH 3: Abemaciclib, as initial therapy for advanced breast cancer. J Clin Oncol 35: 3638-3646, 2017.

8. Draeger E: Clinical profile of glimepiride. Diabetes Res Clin Pract 28: 139-146, 1995.

9. Draeger KE, Wernicke-Panten K, Lomp HJ, Schüler E and Rosskamp R: Long-term treatment of type 2 diabetic patients with the new oral antidiabetic agent glimepiride (Amaryl): A double-blind comparison with glibenclamide. Horm Metab Res 28: 419-425, 1996.

10. Badian M, Korn A, Lehr KH, Malerczyk V and Waldhäusl W: Absolute bioavailability of Glimepiride (Amaryl) after oral administration. Drug Metabol Drug Interact 11: 331-339, 1994.

11. Niemi M, Cascorbi I, Timm R, Kroemer HK, Neuvonen PJ and Kivistö KT: Glyburide and glimepiride pharmacokinetics in subjects with different CYP2C9 genotypes. Clin Pharmacol Ther 72: 326-332, 2002.

12. Otagiri M: Study on binding of drug to serum protein. Yakugaku Zasshi 129: 413-425, 2009 (In Japanese).

13. Meyer MC and Guttman DE: The binding of drugs by plasma proteins. J Pharm Sci 57: 895-918, 1968

14. Jusko WJ and Gretch M: Plasma and tissue protein binding of drugs in pharmacokinetics. Drug Metab Rev 5: 43-140, 1976.

15. Vallner JJ: Binding of drugs by albumin and plasma protein. J Pharm Sci 66: 447-465, 1977. 
16. Langtry HD and Balfour JA: Glimepiride. A review of its use in the management of type 2 diabetes mellitus. Drugs 55: 563-584, 1998.

17. Wang D, Sun Y, Li W, Ye F, Zhang Y, Guo Y, Zhang DY and Suo J: Antiproliferative effects of the CDK6 inhibitor PD0332991 and its effect on signaling networks in gastric cancer cells. Int J Mol Med 41: 2473-2484, 2018.

18. Toogood PL, Harvey PJ, Repine JT, Sheehan DJ, VanderWel SN, Zhou H, Keller PR, McNamara DJ, Sherry D, Zhu T, et al: Discovery of a potent and selective inhibitor of cyclin-dependent kinase 4/6. J Med Chem 48: 2388-2406, 2005.

19. Xu H, Yu S, Liu Q, Yuan X, Mani S, Pestell RG and Wu K: Recent advances of highly selective CDK4/6 inhibitors in breast cancer. J Hematol Oncol 10: 97, 2017.

20. Li B, He H, Tao BB, Zhao ZY, Hu GH, Luo C, Chen JX, Ding XH, Sheng P, Dong Y, et al: Knockdown of CDK6 enhances glioma sensitivity to chemotherapy. Oncol Rep 28: 909-914, 2012.

21. Malumbres M and Barbacid M: Cell cycle, CDKs and cancer: A changing paradigm. Nat Rev Cancer 9: 153-166, 2009.

22. Fujiwara $\mathrm{Y}$, Tamura $\mathrm{K}$, Kondo $\mathrm{S}$, Tanabe $\mathrm{Y}$, Iwasa $\mathrm{S}$, Shimomura A, Kitano S, Ogasawara K, Turner PK, Mori J, et al: Phase 1 study of abemaciclib, an inhibitor of CDK 4 and 6, as a single agent for Japanese patients with advanced cancer. Cancer Chemother Pharmacol 78: 281-288, 2016.

23. Patnaik A, Rosen LS, Tolaney SM, Tolcher AW, Goldman JW, Gandhi L, Papadopoulos KP, Beeram M, Rasco DW, Hilton JF, et al: Efficacy and safety of abemaciclib, an inhibitor of CDK4 and CDK6, for patients with breast cancer, non-small cell lung cancer, and other solid tumors. Cancer Discov 6: 740-753, 2016.

24. Tate SC, Sykes AK, Kulanthaivel P, Chan EM, Turner PK and Cronier DM: A population pharmacokinetic and pharmacodynamic analysis of abemaciclib, in a phase I clinical trial in cancer patients. Clin Pharmacokinet 57: 335-344, 2018

25. Rosenkranz B: Pharmacokinetic basis for the safety of glimepiride in risk groups of NIDDM patients. Horm Metab Res 28: 434-439, 1996.
26. Kacevska M, Robertson GR, Clarke SJ and Liddle C: Inflammation and CYP3A4-mediated drug metabolism in advanced cancer: Impact and implications for chemotherapeutic drug dosing. Expert Opin Drug Metab Toxicol 4: 137-149, 2008.

27. Fahy BN, Guo T and Ghose R: Impact of hepatic malignancy on CYP3A4 gene expression. J Surg Res 178: 768-772, 2012.

28. Rosenkranz B, Profozic V, Metelko Z, Mrzljak V, Lange C and Malerczyk V: Pharmacokinetics and safety of glimepiride at clinically effective doses in diabetic patients with renal impairment. Diabetologia 39: 1617-1624, 1996.

29. Hou L, Zhao T, Liu Y and Zhang Y: Efficacy and safety of sitagliptin compared with sulfonylurea therapy in patients with type 2 diabetes showing inadequately controlled glycosylated hemoglobin with metformin monotherapy: A meta-analysis. Exp Ther Med 9: 1528-1536, 2015.

30. DeFronzo RA: Pharmacologic therapy for type 2 diabetes mellitus. Ann Intern Med 131: 281-303, 1999.

31. Inzucchi SE: Oral antihyperglycemic therapy for type 2 diabetes: Scientific review. JAMA 287: 360-372, 2002.

32. Levey AS, Perrone RD and Madias NE: Serum creatinine and renal function. Annu Rev Med 39: 465-490, 1988.

33. Pun KK, Young RTT, Wang C, Tam CF and Ho PWM: The use of glucagon challenge tests in the diagnostic evaluation of hypoglycemia due to hepatoma and insulinoma. J Clin Endocrinol Metab 67: 546-550, 1988

(i) (2) This work is licensed under a Creative Commons Attribution-NonCommercial-NoDerivatives 4.0 International (CC BY-NC-ND 4.0) License. 